University of Nebraska - Lincoln

DigitalCommons@University of Nebraska - Lincoln

Anthony F. Starace Publications

Research Papers in Physics and Astronomy

$9-1-2001$

\title{
Invariant spinor representations of finite rotation matrices
}

N. L. Manakov

Voronezh State University, manakov@phys.vsu.ru

A. V. Meremianin

Voronezh State University, meremianin@phys.vsu.ru

Anthony F. Starace

University of Nebraska-Lincoln, astarace1@unl.edu

Follow this and additional works at: https://digitalcommons.unl.edu/physicsstarace

Part of the Physics Commons

Manakov, N. L.; Meremianin, A. V.; and Starace, Anthony F., "Invariant spinor representations of finite rotation matrices" (2001). Anthony F. Starace Publications. 81.

https://digitalcommons.unl.edu/physicsstarace/81

This Article is brought to you for free and open access by the Research Papers in Physics and Astronomy at DigitalCommons@University of Nebraska - Lincoln. It has been accepted for inclusion in Anthony F. Starace Publications by an authorized administrator of DigitalCommons@University of Nebraska - Lincoln. 


\title{
Invariant spinor representations of finite rotation matrices
}

\author{
N. L. Manakov and A. V. Meremianin \\ Department of Physics, Voronezh State University, 394693 Voronezh, Russia
}

Anthony F. Starace

Department of Physics and Astronomy, The University of Nebraska, Lincoln, Nebraska 68588-0111

(Received 26 March 2001; published 14 August 2001)

\begin{abstract}
Our recent results [N. L. Manakov et al., Phys. Rev. A 57, 3233 (1998); 61, 022103 (2000)] on the invariant representations of finite rotation matrices (FRM's) of integer rank $j$ (in terms of tensor products of vectors connected with a space-fixed reference frame) are generalized here for the general case of arbitrary (i.e., integer or half-integer) rank $j$. This extension is carried out by using new spinor representations of FRM's in terms of specially introduced spinor-annihilation operators. We demonstrate that all widely used, standard representations of FRM's follow as special cases of our invariant representation for particular parametrizations of the rotation parameters. As the simplest application of invariant spinor representations of FRM's, the factorized form of Wigner $\mathbf{d}^{j}(\beta)$ matrices with an arbitrary rank $j$ is obtained as a product of two triangular matrices composed of various powers of $\cos (\beta / 2)$.
\end{abstract}

DOI: 10.1103/PhysRevA.64.0221XX

PACS number(s): 03.65.Ca, 03.65.Fd

\section{INTRODUCTION}

Finite rotation matrices (FRM's), $R_{k m}^{j}(\Omega)$, are basic objects of the quantum theory of angular momentum (see, e.g., Ref. [1]). We define the FRM $R_{m^{\prime} m}^{j}(\Omega)$ in accordance with the standard relation [1]

$$
\widetilde{T}_{j m}=\sum_{m^{\prime}=-j}^{j} T_{j m^{\prime}} R_{m^{\prime} m}^{j}(\Omega),
$$

where $T_{j m}$ and $\widetilde{T}_{j m}$ are the components of an irreducible tensor $T_{j}$ of rank $j$, given in the "old" (space-fixed) frame $K$ and in the "new" (rotated) frame $\widetilde{K}$, respectively; and $\Omega$ denotes three rotation parameters (e.g., in the case of Wigner $D$ functions [2] they are Euler angles $\alpha, \beta, \gamma)$.

Recently in Ref. [3] (which will henceforth be referred to as I) we introduced the so-called invariant representations of FRM's (i.e., having explicit tensor forms), which are useful, in particular, for analyses of angular distributions in processes involving polarized particles. Our invariant representations are written for symmetrized combinations of $R_{k m}^{j}(\Omega)$, i.e., the "parity-projected" FRM's, which are defined by [4]

$$
\begin{gathered}
R_{k m}^{j \lambda_{p}}(\Omega)=\left(1-\frac{\delta_{0, k}}{2}\right)\left[R_{-k m}^{j}(\Omega)+(-1)^{\left.k+\lambda_{p} R_{k m}^{j}(\Omega)\right]}\right. \\
k \geqslant \lambda_{p}
\end{gathered}
$$

where $\lambda_{p}=0\left(\lambda_{p}=1\right)$ for even (odd) parity. Parity-projected FRM's are closely related with the "real" representations of FRM's [5]. Moreover, parity-projected Wigner $D$ functions (or $\mathbf{d}^{j}(\beta)$ matrices) naturally appear in three-body problems having definite parity (see, e.g., Refs. [6] and [7]).

It was shown that parity-projected FRM's can be expressed in terms of the linear combination of tensor products of two spherical harmonics (bipolar harmonics) depending on two vectors, $\mathbf{n}$ and $\mathbf{n}^{\prime}$, connected with the fixed frame $K$. In I several alternative forms for FRM's were obtained for special choices of the vectors $\mathbf{n}$ and $\mathbf{n}^{\prime}$. The most general result may be presented as [cf. Eq. (7) in Ref. [4]]

$$
R_{k m}^{j \lambda_{p}}(\Omega)=\sum_{s=0}^{k-\lambda_{p}} A_{k s}^{\lambda_{p}}(\theta)\left\{Y_{j-s}(\mathbf{n}) \otimes Y_{s+\lambda_{p}}\left(\mathbf{n}^{\prime}\right)\right\}_{j m},
$$

where $A_{k s}^{\lambda_{p}}(\theta)$ are numerical coefficients dependent on the angle $\theta$ between the vectors $\mathbf{n}$ and $\mathbf{n}^{\prime}(0<\theta<\pi)$ [4], and where the tensor product,

$$
\begin{aligned}
& \left\{Y_{j-s}(\mathbf{n}) \otimes Y_{s+\lambda_{p}}\left(\mathbf{n}^{\prime}\right)\right\}_{j m} \\
& \quad=\sum_{q, q^{\prime}} C_{j-s q s+\lambda_{p} q^{\prime}}^{j m} Y_{j-s q}(\mathbf{n}) Y_{s+\lambda_{p} q^{\prime}}\left(\mathbf{n}^{\prime}\right),
\end{aligned}
$$

is the so-called "minimal bipolar harmonic" $(\mathrm{MBH})$, where $C_{a \alpha b \beta}^{c \gamma}$ is a Clebsh-Gordan coefficient. The vector $\mathbf{n}$ is directed along the $z$ axis of the fixed frame $K$ and the vector $\mathbf{n}^{\prime}$ lies in the $z x$ plane. [Thus the angle $\theta$ is the free parameter, and the three independent real parameters of the rotation $\Omega$ in our approach are determined by the angular coordinates of $\mathbf{n}$ and $\mathbf{n}$ ' in the "new" (or rotated) frame $\widetilde{K}$.] The term "invariant" for the representation of FRM's in the form (3) means that the entire dependence of the FRM $R_{m^{\prime} m}^{j}(\Omega)$ on the tensor index $m$ is concentrated only in the tensor projection $m$ of the MBH's on the right-hand side (rhs) of Eq. (3). [The tensor sense of the index $m$ is obvious from Eq. (1), which may be interpreted as the expansion of a tensor $\widetilde{T}_{j m}$ (in the rotated frame) in $(2 j+1)$ tensors $R_{m^{\prime} m}^{j}(\Omega)$ enumerated by the (nontensor) index $m^{\prime}$.] We use the term "minimal bipolar harmonics" for the parity-projected tensor products in Eq. (4) with index $s=0,1, \ldots, j-\lambda_{p}$, since they form the (minimal) basis set of $(2 j+1)$ irreducible tensors in a space of tensors with an integer rank $j$. [There are $(j$ 
$+1)$ polar tensors for $\lambda_{p}=0$ and $j$ axial tensors (pseudotensors) for $\lambda_{p}=1$.] This fact is obvious, since an arbitrary tensor can be presented as a combination of FRM's [see Eq. (1)], and each of these FRM's has expansion (3) in terms of the set of MBH. The complete basis set of MBH was introduced for the reduction of bipolar harmonics of rank $j$ involving internal tensors of higher ranks than $j$ [8]. These results provide great simplifications for the analysis of angular distributions in reactions involving polarized particles and in $\gamma-2 e$ processes (see, e.g., Refs. [8] and [9]). They are also useful in the analysis of other physical problems (see, e.g., Refs. [1] and [10]).

Our derivations of invariant results for FRM's in I were based on the vector differentiation technique [8], and hence they are valid only for irreducible tensors with integer ranks $j$. In this paper we generalize these results and present invariant representations for FRM's which are valid for an arbitrary (either integer or half integer) rank $j$. This generalization is performed in Sec. III based on specially introduced "spinor-annihilation" $(\hat{\delta})$ operators (Sec. II), which are spinor analogs of the vector $\nabla$ operators. The use of these $\hat{\delta}$ operators leads to the main result of this paper, i.e., the "differential" spinor representation of the FRM [in Eq. (21)] and explicit forms of FRM's in Eqs. (23) and (26), which are valid for both integer and half integer ranks $j$. In Sec. IV we demonstrate the reduction of our spinor representation for the FRM to the known invariant form for the case of integer $j$. Based on the invariant spinor representation, is possible to obtain in a convenient way many fundamental results of angular momentum algebra. In particular, this representation permits the analysis of some interesting algebraic properties of standard Wigner $D$ functions:

$$
D_{k m}^{j}(\alpha, \beta, \gamma)=\exp (-i k \alpha) d_{k m}^{j}(\beta) \exp (-i m \gamma) .
$$

Namely, in Ref. [4] it was found that, for integer rank $j$, the parity-projected $\mathbf{d}^{j}(\beta)$ matrix $\mathbf{d}^{j \lambda_{p}}(\beta)$ can be presented as a product of two triangular matrices composed of Gegenbauer polynomials $C_{n}^{\alpha}(\cos \beta)$ having positive and negative upper indices $\alpha$, respectively. Based on the invariant spinor form of the FRM, in Sec. V we obtain the factorized form of the standard (not "parity-projected") $\mathbf{d}^{j}(\beta)$ matrix with an arbitrary $j$ as a product of two triangular matrices composed of various powers of $\cos (\beta / 2)$. Finally, all known standard parametrizations of FRM's may be obtained as special cases of our invariant results for particular parametrizations of the rotation parameters $\Omega$. As an example, in Sec. VI we demonstrate the reduction of the "differential" spinor representation of the FRM in Eq. (21) to explicit expressions for the FRM in Euler's parametrization [2] as well as in the $(\mathbf{n}, \omega)$ parametrization involving the direction of the rotation axis $\mathbf{n}$ and the rotation angle $\omega[11,12]$.

\section{TENSOR PRODUCTS OF IDENTICAL SPINORS AND SPINOR-ANNIHILATION OPERATORS}

Below we shall obtain an invariant representation of $R_{k m}^{j}(\Omega)$ with arbitrary rank $j$ (either integer or half integer).
It is obvious that to generalize the results valid for spherical tensors with integer ranks to the case of half integer $j$ one needs to use the spinor formalism. Let us first introduce some spinor algebra notations. We use below the Greek letter $\chi$ for a spinor as such, while its components we denote as $\chi_{\mu}, \mu= \pm 1 / 2$. We use in this paper the following definition for the scalar product of irreducible tensors $A_{j}$ and $B_{j}$ in terms of the standard irreducible tensor product $\left\{A_{j} \otimes B_{j}\right\}_{\mathrm{km}}$ [1]:

$$
\left(A_{j} \cdot B_{j}\right)=\sqrt{2 j+1}\left\{A_{j} \otimes B_{j}\right\}_{00} .
$$

Thus the scalar product of spinors $\chi$ and $\phi$ is

$$
(\chi \cdot \phi)=\chi_{1 / 2} \phi_{-1 / 2}-\chi_{-1 / 2} \phi_{1 / 2} .
$$

In particular, $(\chi \cdot \chi)=0$. An arbitrary spinor $\chi$ can be expanded in two base spinors, $\beta^{(+1 / 2)}$ and $\beta^{(-1 / 2)}$, as follows:

$$
\chi=\beta^{(+1 / 2)} \chi_{-1 / 2}-\beta^{(-1 / 2)} \chi_{1 / 2}, \quad \chi_{ \pm 1 / 2}=\left(\chi \cdot \beta^{( \pm 1 / 2)}\right),
$$

where the orthonormalization properties of the base spinors $\beta^{( \pm 1 / 2)}$ are

$$
\left(\beta^{(\alpha)} \cdot \beta^{\left(\alpha^{\prime}\right)}\right)=(-1)^{1 / 2-\alpha} \delta_{\alpha,-\alpha^{\prime}}, \quad \beta_{\mu}^{(\alpha)}=(-1)^{1 / 2-\alpha} \delta_{\mu,-\alpha} .
$$

Note that for simpler presentation of the results below, our definition for the components of spinors $\beta^{( \pm 1 / 2)}$ differs from the standard definition [1] of components of spin-1/2 functions $\chi_{(1 / 2) m}$ with $m= \pm 1 / 2$.

We use the notation $\{\chi\}_{a \alpha}$ for the irreducible tensor of rank $a$ and component $\alpha$ which is the tensor product of $2 a$ spinors $\chi$,

$$
\{\chi\}_{a \alpha}=\left\{\cdots\{\chi \otimes \chi\}_{1} \cdots \otimes \chi\right\}_{a \alpha} .
$$

This definition is similar to that introduced for the case of identical rank-1 tensors (vectors) in Ref. [8] (see also Refs. [13] and [14]) where a number of general properties of such tensor products are discussed. In particular, the tensor product (8) does not depend on the coupling scheme of spinors $\chi$ in accordance with the identity

$$
\left\{R_{a} \otimes\left\{\chi \otimes T_{b}\right\}_{b+1 / 2}\right\}_{a+b+1 / 2}=\left\{\left\{R_{a} \otimes \chi\right\}_{a+1 / 2} \otimes T_{b}\right\}_{a+b+1 / 2},
$$

which can be proved using the tensor recoupling rules [1]. Inserting Eq. (6) into Eq. (8), we obtain

$$
\begin{aligned}
\{\chi\}_{a \alpha}= & \sum_{n=0}^{2 a}\left(\begin{array}{c}
2 a \\
n
\end{array}\right)\left(\chi_{-1 / 2}\right)^{n}\left(-\chi_{1 / 2}\right)^{2 a-n}\left\{\left\{\beta^{(+1 / 2)}\right\}_{n / 2}\right. \\
& \left.\otimes\left\{\beta^{(-1 / 2)}\right\}_{a-n / 2}\right\}_{a \alpha},
\end{aligned}
$$

where $\left(\begin{array}{l}a \\ b\end{array}\right)$ is the binomial coefficient. The tensor product of $\beta^{( \pm 1 / 2)}$ spinors is easily calculated using Eq. (7) and the relation $C_{a a b b}^{a+b a+b}=1$ : 


$$
\left\{\beta^{( \pm 1 / 2)}\right\}_{a \alpha}=( \pm 1)^{2 a} \delta_{\alpha, \mp a} .
$$

Finally, using Eqs. (10) and (11) as well as known analytical results for Clebsh-Gordan coefficients [1], we obtain the following explicit expression for $\{\chi\}_{a \alpha}$ in terms of spinor components $\chi_{\mu}$ :

$$
\{\chi\}_{a \alpha}=\sqrt{\frac{(2 a) !}{(a-\alpha) !(a+\alpha) !}}\left(\chi_{1 / 2}\right)^{a+\alpha}\left(\chi_{-1 / 2}\right)^{a-\alpha} .
$$

The key aspect of our further considerations is our use of "spinor-annihilation operators," $\hat{\delta}$. We introduce such operators in an invariant way,

$$
(\phi \cdot \hat{\delta}) \chi_{\mu}=\phi_{\mu}
$$

where $\phi$ and $\chi$ are arbitrary spinors. In terms of spinor components, the definition (13) is equivalent to the following:

$$
\hat{\delta}_{\nu} \chi_{\mu}=(-1)^{1 / 2+\nu} \delta_{\nu,-\mu},
$$

or, more generally,

$$
\begin{gathered}
\hat{\delta}_{\nu}\left(\chi_{\mu}\right)^{k}=(-1)^{1 / 2+\nu} k\left(\chi_{\mu}\right)^{k-1} \delta_{\nu,-\mu}, \\
\hat{\delta}_{\nu} \chi_{\mu} \chi_{\rho}=(-1)^{1 / 2+\nu}\left(\chi_{\mu} \delta_{\nu,-\rho}+\chi_{\rho} \delta_{\nu,-\mu}\right),
\end{gathered}
$$

where $\delta_{\alpha, \beta}$ is the Kronecker symbol. It is seen from these equations that the spinor operators $\hat{\delta}$ annihilate the spinors $\chi$.

Using Eqs. (12) and (15) one may verify two important relations involving tensor products of $\hat{\delta}$ operators, $\{\hat{\delta}\}_{j m}$ :

$$
\begin{aligned}
\{\hat{\delta}\}_{j m}\{\chi\}_{j k} & =(-1)^{j-k}(2 j) ! \delta_{m,-k}, \\
\left\{\{\hat{\delta}\}_{j} \otimes\{\chi\}_{j}\right\}_{a \alpha} & =\sum_{m, k} C_{j m j k}^{a \alpha}\{\hat{\delta}\}_{j m}\{\chi\}_{j k} \\
& =(-1)^{2 j} \sqrt{2 j+1}(2 j) ! \delta_{a, 0} .
\end{aligned}
$$

In particular, $\{\hat{\delta} \otimes \chi\}_{a \alpha}=-\sqrt{2} \delta_{a, 0}$. We note also the simple relation

$$
(\hat{\delta} \cdot \hat{\delta})\{\chi\}_{j m}=0,
$$

which is obvious from the definition of the scalar product in Eq. (7) and also because (after the annihilation of two spinors $\chi$ ) a tensor having rank $j$ cannot be composed of $2 j-2$ spinors $\chi$. Thus the spinor-annihilation operator $\hat{\delta}$ in the space of tensors composed of spinors $\chi$ may be considered as the spinor generalization (valid for any $j$ ) of the vector gradient operator, $\nabla$, operating in the space of tensors composed of vectors $\mathbf{r}$. This analogy is supported by the comparison of the definition (13) with the vector identity, $\left(\mathbf{a} \cdot \nabla_{\mathbf{c}}\right) c_{k}=a_{k}$. Moreover, the identity (18) with $\hat{\delta} \rightarrow \nabla$ and $\chi \rightarrow \mathbf{r}$ is also valid since the tensor product $\{\mathbf{r}\}_{j m}$ obeys the Laplace equation. One additional property of $\hat{\delta}$ operators that is similar to that for ordinary differential operators and that is useful in concrete applications is given in Eq. (A1) in the Appendix.

\section{SPINOR REPRESENTATIONS OF FRM}

To obtain the representation of FRM in terms of the spinor constructions introduced in the previous section, let us consider the scalar product $\left(T_{j} \cdot\{\chi\}_{j}\right)$, where $T_{j}$ is an arbitrary tensor of rank $j$ (either integer or half integer). In view of the invariance of the scalar product, we have

$$
\left(\widetilde{T}_{j} \cdot\{\tilde{\chi}\}_{j}\right)=\left(T_{j} \cdot\{\chi\}_{j}\right)
$$

where the superscript tilde means that components of $\tilde{\chi}$ and $\widetilde{T}_{j}$ are defined in the rotated coordinate frame $\widetilde{K}$. Acting on both sides of this equation with the operator $\{\tilde{\hat{\delta}}\}_{j m}$ (where $\tilde{\hat{\delta}}$ is the spinor-annihilation operator in the frame $\widetilde{K}$ ), we obtain

$$
\begin{aligned}
& \{\tilde{\hat{\delta}}\}_{j m} \sum_{q=-j}^{j}(-1)^{j-q} \widetilde{T}_{j q}\{\tilde{\chi}\}_{j-q} \\
& \quad=\{\tilde{\tilde{\delta}}\}_{j m} \sum_{k=-j}^{j}(-1)^{j-k} T_{j k}\{\chi\}_{j-k} .
\end{aligned}
$$

Since $\tilde{\hat{\delta}}$ and $\tilde{\chi}$ are defined in the same frame $\widetilde{K}$, we can explicitly calculate the left-hand side (lhs) of Eq. (20) using Eq. (16) (assuming $\tilde{\hat{\delta}}$ does not act on $\widetilde{T}_{k q}$ ). Then after the comparison of the result with the definition of the FRM in Eq. (1) we obtain the "operator representation" of the FRM:

$$
R_{k m}^{j}(\Omega)=\frac{(-1)^{j+k}}{(2 j) !}\{\tilde{\hat{\delta}}\}_{j m}\{\chi\}_{j-k} .
$$

Note that the tensor product of $\tilde{\hat{\delta}}$ operators in this equation must be calculated in the rotated frame $\widetilde{K}$, while the product of spinors $\chi$ is defined in the "old" frame $K$ [and thus the result in Eq. (16) is not applicable here]. We use the term "invariant representation of the FRM" for the result on the rhs of Eq. (21) because it depends on the projections $m$ and $k$ only through invariant tensors and does not depend explicitly on the rotation parameters. Equation (21) is the spinor analog of the invariant "differential" representation of the FRM in the tensor form [see Eq. (24) in I]:

$$
R_{k m}^{j}(\Omega)=\frac{(-1)^{k}}{j !}\{\tilde{\nabla}\}_{j m}\{\mathbf{r}\}_{j-k}, \quad j=0,1,2, \ldots .
$$

An explicit form of the invariant representation of FRM in terms of tensor products of base spinors $\beta^{(\alpha)}$ of the old frame $K$ can be derived after the substitution of Eq. (12) into Eq. (21). In view of the invariance of a scalar product, the components $\chi_{ \pm 1 / 2}$ of the spinor $\chi$ in the frame $K$, which enter from Eq. (12), may be written as the scalar products $\left(\tilde{\chi} \cdot \beta^{(+1 / 2)}\right)$ [cf. Eq. (6)], calculated in the rotated frame $\widetilde{K}$. Thus we have 


$$
\begin{aligned}
R_{k m}^{j}(\Omega)= & \frac{(-1)^{j+k}}{\sqrt{(2 j) !(j-k) !(j+k) !}} \\
& \times\{\tilde{\hat{\delta}}\}_{j m}\left(\tilde{\chi} \cdot \beta^{(+1 / 2)}\right)^{j-k}\left(\tilde{\chi} \cdot \beta^{(-1 / 2)}\right)^{j+k},
\end{aligned}
$$

where the components of all spinors $\tilde{\hat{\delta}}, \tilde{\chi}$, and $\beta^{( \pm 1 / 2)}$ must be calculated in the same frame $\widetilde{K}$. Note that one must distinguish between $\beta^{( \pm 1 / 2)}$ and $\widetilde{\beta}^{( \pm 1 / 2)}$, which are the base spinors of the frames $K$ and $\widetilde{K}$, respectively. Obviously, for the components of $\beta^{( \pm 1 / 2)}$ in the $\widetilde{K}$ frame Eqs. (7) are not valid. Below in the text (unless otherwise specified) we will use the notation $\beta^{( \pm 1 / 2)}$ for the components of spinors $\beta^{( \pm 1 / 2)}$ given in the $\widetilde{K}$ frame. The calculation of the rhs of Eq. (22) is straightforward but quite lengthy. The key steps are as follows: (i) One uses an equation similar to that for $\chi$ in Eq. (6) to expand $\beta^{( \pm 1 / 2)}$ in terms of the base spinors $\widetilde{\beta}^{( \pm 1 / 2)}$ of $\widetilde{K}$; (ii) Using the binomial theorem, one expands the rhs of Eq. (22) in powers of $\tilde{\chi}_{ \pm 1 / 2}$ [cf. Eq. (6)]; (iii) One uses Eq. (12) to expand $\{\widetilde{\hat{\delta}}\}_{j m}$ in powers of $\widetilde{\hat{\delta}}_{\nu}$; (iv) One uses Eq. (15) to calculate the result of the operators $\tilde{\hat{\delta}}_{\nu}$ acting on the components $\tilde{\chi}_{ \pm 1 / 2} ;(\mathrm{v})$ One uses an equation similar to Eq. (12) to express powers of the components of $\beta^{( \pm 1 / 2)}$ on the base spinors $\widetilde{\beta}^{( \pm 1 / 2)}$ in terms of a sum (over $Q$ ) of $\left\{\beta^{(+1 / 2)}\right\}_{a \alpha}[$ where $a=(j-k) / 2$ and $\alpha=Q]$ and $\left\{\beta^{(-1 / 2)}\right\}_{b \beta}$ [where $b=(j+k) / 2$ and $\beta=m-Q$ ]; (vi) One notices that the coefficient of each term in $Q$ is proportional to the Clebsch-Gordan coefficient $C_{a \alpha b \beta}^{j m}$; (vii) Using the definition of a tensor product [cf. Eq. (4)] one may express the rhs of Eq. (22) finally in terms of the following jm tensor:

$$
R_{k m}^{j}(\Omega)=C_{j k}\left\{\left\{\beta^{(-1 / 2)}\right\}_{(j+k) / 2} \otimes\left\{\beta^{(+1 / 2)}\right\}_{(j-k) / 2}\right\}_{j m},
$$

where

$$
C_{j k}=(-1)^{j+k} \sqrt{\frac{(2 j) !}{(j+k) !(j-k) !}},
$$

and where $\left\{\beta^{(+1 / 2)}\right\}_{p \kappa}$ (or $\left\{\beta^{(-1 / 2)}\right\}_{p \kappa}$ ) is the tensor product (8) of $2 p$ spinors $\beta^{(+1 / 2)}$ (or $\beta^{(-1 / 2)}$ ), whose components should be calculated in the (rotated) coordinate frame $\widetilde{K}$. Obviously, these components are connected with standard components $\beta_{\mu}^{(\alpha)}$ [see Eq. (7)] in the "old" coordinate frame $K$. Moreover, this connection determines implicitly the dependence of the rhs of Eq. (23) on the rotation parameters $\Omega$. An alternative derivation of the key result in Eq. (23) is presented in the Appendix.

A more general representation of the FRM than that given in Eq. (23) may be derived if we rewrite the identity (6) as follows:

$$
\beta^{(-1 / 2)}=-\frac{1}{b}\left(a \beta^{(+1 / 2)}+\tilde{\chi}\right),
$$

where $a \equiv-\left(\chi \cdot \beta^{(-1 / 2)}\right)$ and $b \equiv\left(\chi \cdot \beta^{(+1 / 2)}\right)$. (The scalars $a$ and $b$ can be calculated in an arbitrary coordinate frame.) Since the tensor product $\left\{\beta^{(-1 / 2)}\right\}_{(j+k) / 2}$ does not depend on the coupling scheme of spinors $\beta^{(-1 / 2)}$, we find

$$
\begin{aligned}
\left\{\beta^{(-1 / 2)}\right\}_{(j+k) / 2}= & \left(-\frac{a}{b}\right) \sum_{n=0}^{j+k j+k}\left(\begin{array}{c}
j+k \\
n
\end{array}\right) \frac{1}{a^{n}}\left\{\{\tilde{\chi}\}_{n / 2}\right. \\
& \left.\otimes\left\{\beta^{(+1 / 2)}\right\}_{(j+k-n) / 2}\right\}_{(j+k) / 2} .
\end{aligned}
$$

We used in the above derivation the usual binomial formula. Inserting Eq. (25) into the rhs of Eq. (23), after some algebra we obtain the representation of the FRM containing free parameters - the components $a$ and $b$ of the (arbitrary) spinor $\chi$ in the coordinate frame $K$ :

$$
\begin{aligned}
R_{k m}^{j}(\Omega)= & \left(\frac{a}{b}\right)^{j+k} \sqrt{\frac{(2 j) !}{(j-k) !(j+k) !}} \sum_{n=0}^{j+k}\left(\begin{array}{c}
j+k \\
n
\end{array}\right) \frac{1}{a^{n}}\left\{\{\tilde{\chi}\}_{n / 2}\right. \\
& \left.\otimes\left\{\beta^{(+1 / 2)}\right\}_{j-n / 2}\right\}_{j m} .
\end{aligned}
$$

Note that the tensor product on the rhs of this equation should be calculated in the rotated frame $\widetilde{K}$ [cf. Eq. (23)]. The new representations of the FRM with an arbitrary rank $j$ in Eqs. (23) and (26) together with the formal "differential" representation in Eq. (21) are our main results. All of these representations have an explicitly invariant tensor form since their entire dependence on the tensor index $m$ is concentrated only in the tensor projection on the rhs of Eqs. (21), (23), and (26).

The result in Eq. (26) together with the transformation rule in Eq. (1) proves that an arbitrary irreducible tensor of either integer or half integer rank $j$ can be expanded on the basis of $(2 j+1)$ "minimal" tensor products of the kind $\left\{\{\varphi\}_{n / 2} \otimes\{\chi\}_{j-n / 2}\right\}_{j m}$ (with $n=0,1,2, \ldots, 2 j$ ), where $\varphi$ and $\chi$ are (in general, arbitrary) spinors. In I we have shown that the set of minimal bipolar harmonics, Eq. (4), can also serve as a basis in the space of irreducible tensors of integer ranks. We do not present here the explicit connection between these two bases for the case of integer $j$, although it may be easily derived considering the transformation of the $\mathrm{MBH}$ under the rotation $\Omega$ with the use of the spinor representation (26) for the FRM in Eq. (1).

\section{CONNECTION TO THE CASE OF INTEGER $\boldsymbol{j}$}

In order to obtain the connection between invariant spinor representations of FRM and the representations in vector form presented in I, we transform Eq. (23) to the form

$$
\begin{gathered}
R_{k m}^{j}(\Omega)=C_{j k}\left\{\left\{\left\{\beta^{(+1 / 2)} \otimes \beta^{(-1 / 2)}\right\}_{1}\right\}_{j-k} \otimes\left\{\beta^{(-1 / 2)}\right\}_{k}\right\}_{j m}, \\
k \geqslant 0,
\end{gathered}
$$

where we have used the fact that the tensor product on the 
rhs of Eq. (27) does not depend on the coupling scheme for internal tensors. Note that for integer $k$ the tensor product $\left\{\beta^{(-1 / 2)}\right\}_{k \kappa}$ may be written as [cf. Eqs. $\left.(8,9)\right]$

$$
\begin{aligned}
\left\{\beta^{(-1 / 2)}\right\}_{k \kappa}= & \left\{\{ \beta ^ { ( - 1 / 2 ) } \otimes \beta ^ { ( - 1 / 2 ) } \} _ { 1 } \otimes \cdots \otimes \left\{\beta^{(-1 / 2)}\right.\right. \\
& \left.\left.\otimes \beta^{(-1 / 2)}\right\}_{1}\right\}_{k \kappa} .
\end{aligned}
$$

Obviously, components of the tensor product of two spinors having the rank 1 are spherical components of a vector, $\mathbf{v}$ $\equiv\left\{\beta^{(+1 / 2)} \otimes \beta^{(-1 / 2)}\right\}_{1}$. The components of this vector in the frame $K$ may be easily calculated taking into account the identities in Eq. (7),

$$
v_{0}=-C_{\frac{1}{2}}^{10}-\frac{1}{2} \frac{1}{2} \frac{1}{2}=-\frac{1}{\sqrt{2}}, \quad v_{ \pm 1}=0 .
$$

Thus, we obtain that $\mathbf{v}=-\mathbf{e}_{0} / \sqrt{2}$, where $\mathbf{e}_{0}$ is the unit vector directed along the axis $\mathrm{Z}$ of the frame $K$. Similarly, in the $K$ frame we have following relations:

$$
\left\{\beta^{(-1 / 2)} \otimes \beta^{(-1 / 2)}\right\}_{11}=C_{\frac{1}{2} \frac{1}{2} \frac{1}{2} \frac{1}{2}}^{11}=1,
$$

while the components $\left\{\beta^{(-1 / 2)} \otimes \beta^{(-1 / 2)}\right\}_{1 \nu}$ with $\nu=0$ and $\nu$ $=-1$ are zero. Therefore we obtain another vector identity, $\left\{\beta^{(-1 / 2)} \otimes \beta^{(-1 / 2)}\right\}_{1}=-\mathbf{e}_{-1}$, where $\mathbf{e}_{-1}$ and $\mathbf{e}_{+1}$ are the spherical unit tensors in the frame $K$. Thus, for integer values of $j$, Eq. (27) can be reduced to the known result for the FRM in terms of basis vectors of the "old" frame $K$ [cf. Eq. (17) in I]:

$$
R_{k m}^{j}(\Omega)=A_{j k}\left\{\left\{\mathbf{e}_{-1}\right\}_{k} \otimes\left\{\mathbf{e}_{0}\right\}_{j-k}\right\}_{j m}, \quad k \geqslant 0,
$$

where the coefficients $A_{j k}$ are related to the $C_{j k}$ in Eq. (23):

$$
A_{j k}=(-1)^{j}(\sqrt{2})^{k-j} C_{j k}=(-1)^{k} \sqrt{\frac{2^{k-j}(2 j) !}{(j-k) !(j+k) !}} .
$$

Obviously, in a similar analysis for the case of half integer $j$ we will have one "uncompensated" spinor $\beta^{(\nu)}$ in the tensor product (27).

\section{FACTORIZED FORM OF WIGNER MATRICES}

Invariant spinor representations of FRM's may prove to be useful in various applications involving angular momentum algebra, especially those in which the tensor structure of the FRM provides more insight into the underlying physics of a process than does its explicit algebraic expression (e.g., as in the analysis of spin polarization effects). In addition, they also permit one to derive new representations of FRM's. As an example, we obtain below a new factorized form of Wigner functions for arbitrary values of $j$; this is similar to the results obtained in Ref. [4] (see also Ref. [7]) for parityprojected matrices having integer $j$. We note first that Eq. (26) can be considered as a product of two triangular matrices. The explicit form of these matrices for the case of Euler's parametrization of the FRM, i.e., for Wigner $D$ functions Eq. (5), can be obtained by considering the special case in which the spinor $\tilde{\chi}$ in Eq. (26) is the base spinor $\widetilde{\beta}^{(+1 / 2)}$ of the rotated frame $\widetilde{K}$. For this case, coefficients $a$ and $b$ (which are "coordinates" of the spinor $\chi \equiv \widetilde{\beta}^{(+1 / 2)}$ in the $K$ frame) are connected with the Euler angles $\alpha, \beta, \gamma$ as follows:

$$
\begin{aligned}
a & =-\left(\widetilde{\beta}^{(+1 / 2)} \cdot \beta^{(-1 / 2)}\right)=D_{(1 / 2)(1 / 2)}^{1 / 2}(\alpha \beta \gamma) \\
& =e^{-i(\alpha+\gamma) / 2} \cos (\beta / 2) \\
b & =\left(\widetilde{\beta}^{(+1 / 2)} \cdot \beta^{(+1 / 2)}\right)=D_{-(1 / 2)(1 / 2)}^{1 / 2}(\alpha \beta \gamma) \\
& =e^{i(\alpha-\gamma) / 2} \sin (\beta / 2)
\end{aligned}
$$

These identities follow immediately from Eqs. (1) and (6). The calculation in the $\widetilde{K}$ frame of the tensor product on the rhs of Eq. (26) can be performed explicitly by standard angular momentum algebra [1]:

$$
\begin{aligned}
&\left\{\left\{\widetilde{\beta}^{(+1 / 2)}\right\}_{n / 2} \otimes\left\{\beta^{(+1 / 2)}\right\}_{j-n / 2}\right\}_{j m} \\
&= C_{n / 2,-n / 2, j-n / 2,(m+n / 2)}^{j m} \sqrt{\frac{(2 j-n) !}{(j+m) !(j-m-n) !}} \\
& \times\left(\beta^{(+1 / 2)} \cdot \widetilde{\beta}^{(+1 / 2)}\right)^{j+m}\left(\beta^{(+1 / 2)} \cdot \widetilde{\beta}^{(-1 / 2)}\right)^{j-m-n} .
\end{aligned}
$$

Here we have used Eqs. (11) and (12) for the calculation of $\left\{\widetilde{\beta}^{(+1 / 2)}\right\}_{n / 2}$ and $\left\{\beta^{(+1 / 2)}\right\}_{j-n / 2}$, respectively.

Replacing the Clebsh-Gordan coefficient on the rhs of Eq. (30) by its analytic expression [1] and then inserting Eq. (30) [with the account of Eq. (29)] into Eq. (26), we obtain (upon omitting the trivial dependence of the $D$ functions on the angles $\alpha$ and $\gamma$ ) the following expression for the $d_{k m}^{j}(\beta)$ matrix:

$$
\begin{aligned}
d_{k m}^{j}(\beta)= & \sqrt{\frac{(j-m) !(j+k) !}{(j+m) !(j-k) !}}\left(\sin \frac{\beta}{2}\right)^{m-k} \sum_{n=0}^{j_{m}}(-1)^{n} \\
& \times \frac{(2 j-n) !}{n !(j+k-n) !(j-m-n) !}\left(\cos \frac{\beta}{2}\right)^{2 j+k-m-2 n},
\end{aligned}
$$

where $j_{m}=\min (j-m, j+k)$. Eq. (31) can be considered a matrix identity.

For a simpler presentation, it is convenient to introduce a slightly different matrix, $\overline{\mathbf{d}}^{j}$, instead of the standard $\mathbf{d}^{j}$ matrix:

$$
d_{p-j, q-j}^{j}(\beta)=(-1)^{p}\left(\sin \frac{\beta}{2}\right)^{q-p} \sqrt{\frac{p !(2 j-q) !}{q !(2 j-p) !}} \bar{d}_{p q}^{j}(\beta),
$$

where $p, q=0,1,2, \ldots, 2 j$. Thus one may write the matrix identity in Eq. (31) in terms of the $\overline{\mathbf{d}}^{j}$ matrix as a factorized product: 


$$
\overline{\mathbf{d}}^{j}=\mathbf{C} \cdot \mathbf{A},
$$

or, in explicit form,

$$
\bar{d}_{p q}^{j}(\beta)=\sum_{n=0}^{2 j} C_{p n}(\beta) A_{n q}(\beta),
$$

where the matrix elements of the $\mathbf{A}$ and $\mathbf{C}$ matrices are

$$
\begin{gathered}
\mathbf{A} \equiv A_{n q}(\beta)=\left(\begin{array}{c}
2 j-n \\
q
\end{array}\right)\left(\cos \frac{\beta}{2}\right)^{2 j-n-q}, \\
\mathbf{C} \equiv C_{p n}(\beta)=\left(\begin{array}{l}
p \\
n
\end{array}\right)\left(-\cos \frac{\beta}{2}\right)^{p-n} .
\end{gathered}
$$

It is seen from the above equations that the matrix $\mathbf{A}$ is an upper-left matrix, while $\mathbf{C}$ is a lower left one; diagonal elements of both these matrices are equal to 1 . It is an important fact that the matrix elements of the $(2 j+1)$-dimensional matrices $\mathbf{A} \equiv \mathbf{A}^{(k)}$ and $\mathbf{C} \equiv \mathbf{C}^{(k)}$, where $k=2 j+1$, do not depend on the rank $j$, because it enters Eq. (33) only in the combination $(2 j-n)$, which determines the dimension of the matrices. As a consequence, the $\mathbf{A}^{(k+1)}$ (or $\mathbf{C}^{(k+1)}$ ) matrix can be calculated by simply adding one additional highest (or lowest) row to $\mathbf{A}^{(k)}$ (or $\mathbf{C}^{(k)}$ ). As an example, for $j=1$ we have

$$
\begin{aligned}
& \mathbf{A}^{(3)}=\left(\begin{array}{cccc}
\cos ^{2} \frac{\beta}{2} & 2 \cos \frac{\beta}{2} & & 1 \\
\ldots \ldots & \ldots \ldots & & \\
\cos \frac{\beta}{2} & 1 & \vdots & 0 \\
1 & 0 & \vdots & 0
\end{array}\right), \\
& \mathbf{C}^{(3)}=\left(\begin{array}{cccc}
1 & 0 & \vdots & 0 \\
-\cos \frac{\beta}{2} & 1 & \vdots & 0 \\
\ldots \ldots & \ldots \ldots & \vdots & \\
\cos ^{2} \frac{\beta}{2} & -2 \cos \frac{\beta}{2} & & 1
\end{array}\right),
\end{aligned}
$$

where the marked $2 \times 2$ internal matrices in these equations are results for $j=1 / 2$.

The matrix identity (32) can be explicitly inverted, as was done for the parity-projected $\mathbf{d}^{j \lambda_{p}}(\beta)$ matrices [4]. To demonstrate this, it is sufficient to consider the rotation of the tensor product defined by the lhs of Eq. (30) from the $K$ to the $\widetilde{K}$ coordinate frames. In accordance with Eq. (1) we have

$$
\begin{aligned}
& \left\{\left\{\widetilde{\beta}^{(1 / 2)}\right\}_{n / 2} \otimes\left\{\beta^{(1 / 2)}\right\}_{j-n / 2}\right\}_{j m} \\
& \quad=\sum_{k=-j}^{j}\left\{\left\{\widetilde{\beta}^{(1 / 2)}\right\}_{n / 2} \otimes\left\{\beta^{(1 / 2)}\right\}_{(j-n) / 2}\right\}_{j k}^{(K)} D_{k m}^{j}(\alpha \beta \gamma),
\end{aligned}
$$

where the superscript $(K)$ on the rhs of this equation means that the corresponding tensor product should be calculated in the $K$ frame. It is evident that Eq. (34) can be written in matrix form (omitting once again the trivial dependence on $\alpha$ and $\gamma$ ):

$$
\mathbf{A}=\mathbf{B} \cdot \overline{\mathbf{d}}^{j},
$$

or explicitly,

$$
A_{q n}(\beta)=\sum_{p=0}^{2 j} B_{n p}(\beta) \bar{d}_{p q}^{j}(\beta),
$$

where the matrix $\mathbf{B}$ is

$$
\mathbf{B} \equiv B_{n p}=\left(\begin{array}{l}
n \\
p
\end{array}\right)\left(\cos \frac{\beta}{2}\right)^{n-p} .
$$

Comparing Eqs. (35) and (36) with Eq. (32), we obtain an explicit form for the matrix inverse of $\mathbf{C}$ :

$$
\mathbf{C}^{-1} \equiv\left(C^{-1}\right)_{n p}=B_{n p} .
$$

\section{REDUCTION OF THE INVARIANT REPRESENTATION OF THE FRM TO STANDARD RESULTS}

In what follows we shall demonstrate how known standard parametrizations of FRM's may be deduced as special cases of our invariant results. As may be seen from the general identity for the FRM, Eq. (21), in order to reduce this result for the case of a concrete parametrization of the rotation parameters $\Omega$ one needs to use the spinor transformation rule in terms of parameters that describe the chosen parametrization.

For the Wigner parametrization of $\Omega$, the spinor transformation rule in terms of Euler angles may be written as follows [1]:

$$
\begin{aligned}
\chi_{1 / 2} & =e^{i(\alpha / 2)}\left(e^{i(\gamma / 2)} \cos \frac{\beta}{2} \tilde{\chi}_{1 / 2}-e^{-i(\gamma / 2)} \sin \frac{\beta}{2} \tilde{\chi}_{-1 / 2}\right) \\
\chi_{-1 / 2} & =e^{-i(\alpha / 2)}\left(e^{i(\gamma / 2)} \sin \frac{\beta}{2} \tilde{\chi}_{1 / 2}+e^{-i(\gamma / 2)} \cos \frac{\beta}{2} \tilde{\chi}_{-1 / 2}\right),
\end{aligned}
$$

where the notations $\chi_{ \pm 1 / 2}\left(\tilde{\chi}_{ \pm 1 / 2}\right)$ denote the components of the spinor in the "old" ("new") coordinate frame. Upon inserting Eqs. (38) into Eq. (21), taking into account Eq. (12), we obtain (after some simple algebra) the following result: 


$$
\begin{aligned}
R_{m^{\prime} m}^{j}= & e^{-i\left(m^{\prime} \alpha+m \gamma\right)} \frac{(-1)^{2 j}}{\sqrt{(j-m) !\left(j-m^{\prime}\right) !(j+m) !\left(j+m^{\prime}\right) !}} \\
& \times\left(\widetilde{\hat{\delta}}_{1 / 2}\right)^{j+m}\left(\tilde{\hat{\delta}}_{-1 / 2}\right)^{j-m} \sum_{n, n^{\prime}}(-1)^{n}\left(\begin{array}{c}
j-m^{\prime} \\
n
\end{array}\right)\left(\begin{array}{c}
j+m^{\prime} \\
n^{\prime}
\end{array}\right) \\
& \times\left(\tilde{\chi}_{1 / 2}\right)^{n+n^{\prime}}\left(\tilde{\chi}_{-1 / 2}\right)^{2 j-n-n^{\prime}}\left(\cos \frac{\beta}{2}\right)^{n-n^{\prime}+j+m^{\prime}} \\
& \times\left(\sin \frac{\beta}{2}\right)^{n^{\prime}-n+j-m^{\prime}} .
\end{aligned}
$$

The calculation of the action of the spinor annihilation operators in this equation [cf. Eqs. (13) and (14)] gives

$$
\begin{aligned}
R_{m^{\prime} m}^{j}= & e^{-i\left(m^{\prime} \alpha+m \gamma\right)}\left(\cos \frac{\beta}{2}\right)^{m+m^{\prime}}\left(\sin \frac{\beta}{2}\right)^{m-m^{\prime}} \\
& \times \sqrt{\frac{(j+m) !(j-m) !}{\left(j+m^{\prime}\right) !\left(j-m^{\prime}\right) !} \sum_{n}(-1)^{j-m+n}\left(\begin{array}{c}
j-m^{\prime} \\
n
\end{array}\right)} \\
& \times\left(\begin{array}{c}
j+m^{\prime} \\
j-m-n
\end{array}\right)\left(\cos \frac{\beta}{2}\right)^{2 n}\left(\sin \frac{\beta}{2}\right)^{2 j-2 m-2 n}
\end{aligned}
$$

Here the sum over $n$ defines the Jacobi polynomial $P_{k}^{(a, b)}(\cos \beta)$ [15]. Thus we have obtained the standard definition of Wigner's functions in terms of Jacobi polynomials [1]:

$$
\begin{aligned}
R_{m^{\prime} m}^{j}(\alpha \beta \gamma) \equiv & D_{m^{\prime} m}^{j}(\alpha \beta \gamma) \\
= & e^{-i\left(m^{\prime} \alpha+m \gamma\right)} \sqrt{\frac{(j+m) !(j-m) !}{\left(j+m^{\prime}\right) !\left(j-m^{\prime}\right) !}} \\
& \times\left(\cos \frac{\beta}{2}\right)^{m+m^{\prime}}\left(\sin \frac{\beta}{2}\right)^{m-m^{\prime}} \\
& \times P_{j-m}^{\left(m-m^{\prime}, m+m^{\prime}\right)}(\cos \beta) .
\end{aligned}
$$

Besides the Euler angles, another widely used parametrization of the rotation $\Omega$ for which FRM's have a simple analytical form is the $(\mathbf{n}, \omega)$ parametrization, where the unit vector $\mathbf{n}$ defines the rotation axis and $\omega$ is the rotation angle $[11,12]$ (see also Ref. [16]). In this parametrization the transformation rule for a spinor $\chi$ has the form [1]

$$
\chi=\tilde{\chi} \cos \frac{\omega}{2}-i \sqrt{3}\{\mathbf{n} \otimes \tilde{\chi}\}_{1 / 2} \sin \frac{\omega}{2} .
$$

Here we use the notation $\chi$ for the spinor whose components $\chi_{ \pm 1 / 2}$ are defined in the "old" frame $K$ [cf. Eq. (38)]. From Eq. (21), it follows that

$$
R_{m^{\prime} m}^{j}=\frac{1}{(2 j) !} \sum_{a \alpha}(-1)^{j+m^{\prime}} C_{j m j-m^{\prime}}^{a \alpha}\left\{\left\{\{\tilde{\hat{\delta}}\}_{j} \otimes\{\chi\}_{j}\right\}_{a \alpha} .\right.
$$

In order to calculate the tensor product on the rhs of this equation, we note that it is proportional to the spherical harmonic $\bar{Y}_{a \alpha}(\mathbf{n})$,

$$
\left\{\{\tilde{\hat{\delta}}\}_{j} \otimes\{\chi\}_{j}\right\}_{a \alpha}=A_{a} \bar{Y}_{a \alpha}(\mathbf{n}),
$$

where $\bar{Y}_{a \alpha}(\mathbf{n})$ is a "renormalized" spherical harmonic, $\bar{Y}_{a \alpha}(\mathbf{n}) \equiv \sqrt{4 \pi /(2 a+1)} Y_{a \alpha}(\mathbf{n})$. The identity (42) may be understood by considering that after the action of the $\widetilde{\hat{\delta}}$ operators on the spinors $\tilde{\chi}$, taking into account Eq. (40), the tensor of rank $a$ on the lhs of Eq. (42) may be composed only of unit vectors $\mathbf{n}$, whose angular representation is the spherical harmonic $Y_{a \alpha}(\mathbf{n})$. The numerical coefficient $A_{a}$ in Eq. (42) may be calculated in an appropriate coordinate frame, the simplest of which is the frame having its $Z$ axis directed along the vector $\mathbf{n}$. In this coordinate frame the components of $\chi$ follow from Eq. (40):

$$
\chi_{ \pm 1 / 2}=\tilde{\chi}_{ \pm 1 / 2} e^{ \pm i(\omega / 2)} .
$$

Then, calculating the tensor product on the lhs of Eq. (42), we find for the coefficient $A_{a}$ the following chain of equations:

$$
\begin{aligned}
A_{a}= & \sum_{n} C_{j n j-n}^{a 0}\left\{\tilde{\hat{\delta}}_{j n}\{\chi\}_{j-n}\right. \\
= & \sum_{n} C_{j n j-n}^{a 0} \frac{(2 j) !}{(j-n) !(j+n) !}\left(\widetilde{\hat{\delta}}_{1 / 2}\right)^{j+n}\left(\tilde{\hat{\delta}}_{-1 / 2}\right)^{j-n} \\
& \times\left(\tilde{\chi}_{1 / 2}\right)^{j-n}\left(\tilde{\chi}_{-} \frac{1}{2}\right)^{j+n} e^{-i \omega n} \\
= & (2 j) ! \sum_{n}(-1)^{j+n} C_{j n j-n}^{a 0} e^{-i \omega n} \\
= & (-i)^{a}(-1)^{2 j}(2 j) ! \frac{\sqrt{2 a+1}}{\sqrt{2 j+1}} \chi_{a}^{j}(\omega),
\end{aligned}
$$

where the results on the second and third lines follow from Eqs. (12) and (14), respectively, and where $\chi_{a}^{j}(\omega)$ is the generalized character of the rotation group $\mathrm{O}(3)$ (cf. Sec. 4.15 of Ref. [1]). Inserting Eq. (43) into Eq. (42) and then that result into Eq. (41), we obtain

$$
\begin{aligned}
R_{m^{\prime} m}^{j}(\mathbf{n}, \omega) & \equiv U_{m^{\prime} m}^{j}(\mathbf{n}, \omega) \\
& =\sum_{a=0}^{2 j}(-i)^{a} \frac{2 a+1}{2 j+1} \chi_{a}^{j}(\omega) C_{j m^{\prime} a \alpha}^{j m} \bar{Y}_{a \alpha}(\mathbf{n}) .
\end{aligned}
$$

The result in Eq. (44) coincides with the standard definition of the FRM $U_{m^{\prime} m}^{j}(\mathbf{n}, \omega)$ for the case of the $(\mathbf{n}, \omega)$ parametrization $[1,11]$.

We note also the parametrization of $\Omega$ in terms of CayleyKlein parameters, which are two complex numbers $a$ and $b$ normalized by the condition $|a|^{2}+|b|^{2}=1[1,17]$. The con- 
nection of $a$ and $b$ with Euler angles is given by the Wigner functions $D_{(1 / 2)(1 / 2)}^{1 / 2}(\alpha, \beta, \gamma)$ and $D_{-(1 / 2)(1 / 2)}^{1 / 2}(\alpha, \beta, \gamma)$, respectively. It is seen from Eq. (29) that in the case when the "free spinor" $\chi$ coincides with the base spinor $\widetilde{\beta}^{(+1 / 2)}$ of the "new" frame $\widetilde{K}$, its components in the "old" frame $K, a$ and $b$, are nothing else than Cayley-Klein parameters. Moreover, it is possible to write the tensor product in Eq. (30) in terms of $a$ and $b$, thus obtaining the explicit representation of FRM's in terms of Cayley-Klein parameters $[1,17]$. However, we do not present the derivation of this result here, because it is not used widely in physical problems.

\section{CONCLUSION}

This paper completes our analysis of invariant representations of finite rotation matrices (see I and [4]). In Sec. III we have obtained the most general invariant representations of the FRM, as they are valid for both integer and half integer $j$. We have also established the relation of these general spinor representations to both our previous results for integer $j$ (in Sec. IV) and to the standard representations for FRM's (in Sec. VI). Besides applications to the general theory of angular momentum (demonstrated in Sec. V), invariant representations of FRM's are useful in physical problems because they provide a powerful tool for the analysis of general properties of a physical phenomenon based only on symmetry considerations, taking into account the invariant (e.g., vector or spinor) characteristics inherent to the concrete problem. These applications are based on an invariant (i.e., independent of a concrete coordinate frame) analysis of the fundamental mathematical objects of atomic theory, irreducible tensor operators $T_{j m}$. The idea of an invariant parametrization of tensor operators was realized in I, where invariant representations of FRM's were introduced. Then, using the transformation rule (1), $T_{j m}$ (or more exactly, $\widetilde{T}_{j m}$, i.e., the operator $T_{j m}$ in an arbitrary reference frame $\widetilde{K}$ ) may be presented in terms of its $j m^{\prime}$ components in a suitable frame $K$ and of invariant FRM's, without an explicit parametrization of the rotation $\Omega$ [see, e.g., Eqs. (53) and (54) in I for the case of integer $j]$. Moreover, the parameters which (implicitly) describe the rotation $\Omega$ (e.g., two noncollinear vectors or components of the spinor $\chi$ in a fixed coordinate frame) may be connected with some physical quantities inherent to the problem being analyzed. A number of examples have been presented in I. We emphasize only that such invariant methods are especially fruitful for the separation of kinematical (i.e., dependent on the geometry, polarization states, and momentum directions of the target and projectiles) from dynamical factors in cross sections of atomic collisions with photons and/or electrons.

\section{ACKNOWLEDGMENTS}

This work has been supported in part by NSF Grant No. PHY-0070980, and by RFBR Grant No. 00-02-17843 and the Grant Program "Universities of Russia."

\section{APPENDIX: AUXILIARY IDENTITY FOR $\hat{\boldsymbol{\delta}}$ OPERATORS AND ALTERNATIVE DERIVATION OF EQ. (23)}

In applications of the $\hat{\delta}$-operator formalism introduced in Sec. II an auxiliary identity for the product of two homogeneous $n$-linear forms, one of them involving spinor operators $\hat{\delta}$ and another involving spinors $\chi$, may be useful. Consider therefore the following such product:

$$
\begin{aligned}
& \left(\sum_{\mu_{1}, \ldots, \mu_{n}= \pm 1 / 2} a_{\mu_{1}, \ldots, \mu_{n}} \hat{\delta}_{\mu_{1}} \cdots \hat{\delta}_{\mu_{n}}\right) \\
& \quad \times\left(\sum_{\nu_{1}, \ldots, \nu_{n}= \pm 1 / 2} b_{\nu_{1}, \ldots, \nu_{n}} \chi_{\nu_{1}} \cdots \chi_{\nu_{n}}\right) \\
& =(-1)^{n}\left(\sum_{\nu_{1}, \ldots, \nu_{n}= \pm 1 / 2} b_{\nu_{1}, \ldots, \nu_{n}} \hat{\delta}_{\nu_{1}} \cdots \hat{\delta}_{\nu_{n}}\right) \\
& \quad \times\left(\sum_{\mu_{1}, \ldots, \mu_{n}= \pm 1 / 2} a_{\mu_{1}, \ldots, \mu_{n}} \chi_{\mu_{1}} \cdots \chi_{\mu_{n}}\right),
\end{aligned}
$$

where the coefficients $a_{\mu_{1}, \ldots, \mu_{n}}$ and $b_{\nu_{1}, \ldots, \nu_{n}}$ may be composed of spinors other than $\chi$ and $\hat{\delta}$. Equation (A1) is completely equivalent to the similar identity for the case involving ordinary differential operators [18]. The result in Eq. (A1) may be verified by direct calculation as it illustrates the following chain of equations for the case $n=2$ :

$$
\begin{aligned}
\left(\sum_{\mu_{1}, \mu_{2}= \pm 1 / 2} a_{\mu_{1} \mu_{2}} \hat{\delta}_{\mu_{1}} \hat{\delta}_{\mu_{2}}\right)\left(\sum_{\nu_{1}, \nu_{2}= \pm 1 / 2} b_{\nu_{1} \nu_{2}} \chi_{\nu_{1}} \chi_{\nu_{2}}\right) \\
=\sum_{\mu_{1}, \mu_{2}} \sum_{\nu_{1}, \nu_{2}} a_{\mu_{1} \mu_{2}} b_{\nu_{1} \nu_{2}}(-1)^{1+\mu_{1}+\mu_{2}}\left(\delta_{\mu_{1},-\nu_{1}} \delta_{\mu_{2},-\nu_{2}}\right. \\
\left.\quad+\delta_{\mu_{1},-\nu_{2}} \delta_{\mu_{2},-\nu_{1}}\right) \\
=\sum_{\mu_{1}, \mu_{2}} \sum_{\nu_{1}, \nu_{2}} a_{\mu_{1} \mu_{2}} b_{\nu_{1} \nu_{2}} \hat{\delta}_{\nu_{1}} \hat{\delta}_{\nu_{2}} \chi_{\mu_{1}} \chi_{\mu_{2}} \\
=\left(\sum_{\nu_{1}, \nu_{2}} b_{\nu_{1} \nu_{2}} \hat{\delta}_{\nu_{1}} \hat{\delta}_{\nu_{2}}\right)\left(\sum_{\mu_{1}, \mu_{2}} a_{\mu_{1} \mu_{2}} \chi_{\mu_{1}} \chi_{\mu_{2}}\right)
\end{aligned}
$$

where $\delta_{\mu,-\nu}$ is the Kronecker symbol. The first equality in the above chain of equations follows from the definitions of $\hat{\delta}$ 's in Eqs. (14) and (15). In the third line term, the $\hat{\delta}$ and $\chi$ components are introduced again (only with reversed indices) using once again Eq. (14) and the evident symmetry relation

$$
\hat{\delta}_{\nu} \chi_{\mu}=(-1)^{1 / 2+\nu} \delta_{\nu,-\mu}=-\hat{\delta}_{\mu} \chi_{\nu} .
$$

The case of a product of two $n$-linear forms may be analyzed similarly (e.g., for three-linear forms, six terms with products of three Kronecker symbols will appear in the second line, etc). Therefore, in the products of two $n$-linear homogeneous 
forms composed of $\hat{\delta}$ 's and $\chi$ 's one can replace $\hat{\delta} \leftrightarrow-\chi$. [The necessity of the minus sign for odd $n$ is evident from Eq. (A2).]

To demonstrate the utility of Eq. (A1) in concrete calculations involving complicated constructions of spinorannihilation operators, we present an alternative derivation of the basic representation (23) for FRM's. With the use of Eq. (A1), the rhs of Eq. (22) may be written as follows:

$$
\begin{aligned}
& \{\tilde{\tilde{\delta}}\}_{j m}\left(\tilde{\chi} \cdot \beta^{(+1 / 2)}\right)^{j-k}\left(\tilde{\chi} \cdot \beta^{(-1 / 2)}\right)^{j+k} \\
& \quad=(-1)^{2 j}\left(\tilde{\tilde{\delta}} \cdot \beta^{(+1 / 2)}\right)^{j-k}\left(\tilde{\hat{\delta}} \cdot \beta^{(-1 / 2)}\right)^{j+k}\{\tilde{\chi}\}_{j m} .
\end{aligned}
$$

Here the calculation of the term on the rhs of Eq. (A3) is much simpler than the straightforward but lengthy approach described in Sec. III for calculating the lhs. Indeed, taking into account Eq. (13) and the independence of $\{\tilde{\chi}\}_{\mathrm{km}}$ of the coupling scheme of rank-1/2 tensors $\tilde{\chi}$, one obtains

$$
\begin{aligned}
\left(\tilde{\hat{\delta}} \cdot \beta^{(-1 / 2)}\right)^{j+k}\{\tilde{\chi}\}_{j m}= & \left(\tilde{\hat{\delta}} \cdot \beta^{(-1 / 2)}\right)^{j+k-1}(-2 j)\left\{\beta^{(-1 / 2)}\right. \\
& \left.\otimes\{\tilde{\chi}\}_{j-1 / 2}\right\}_{j m} \\
= & \left(\tilde{\hat{\delta}} \cdot \beta^{(-1 / 2)}\right)^{j+k-2} 2 j(2 j-1) \\
& \times\left\{\left\{\beta^{(-1 / 2)}\right\}_{1} \otimes\{\tilde{\chi}\}_{j-1}\right\}_{j m} \\
= & \cdots \\
= & (-1)^{j+k} \frac{(2 j) !}{(j-k) !}\left\{\beta^{(-1 / 2)}\right\}_{(j+k) / 2} \\
& \left.\otimes\{\tilde{\chi}\}_{(j-k) / 2}\right\}_{j m} .
\end{aligned}
$$

Calculating similarly the action of $\left(\tilde{\hat{\delta}} \cdot \beta^{(+1 / 2)}\right)^{j-k}$ on $\{\tilde{\chi}\}_{(j-k) / 2}\left[\right.$ which gives $\left.(-1)^{j-k}(j-k) !\left\{\beta^{(+1 / 2)}\right\}_{(j-k) / 2}\right]$ and inserting the results in Eq. (22), we obtain Eq. (23) once again.
[1] D. A. Varshalovich, A. N. Moskalev, and V. K. Khersonskii, Quantum Theory of Angular Momentum (World Scientific, Singapore, 1988).

[2] E. P. Wigner, Group Theory and Its Application to the Quantum Mechanics of Atomic Spectra (Academic Press, New York, 1959).

[3] N. L. Manakov, A. V. Meremianin, and A. F. Starace, Phys. Rev. A 57, 3233 (1998).

[4] N. L. Manakov, A. V. Meremianin, and A. F. Starace, Phys. Rev. A 61, 022103 (2000).

[5] U. Fano, J. Math. Phys. 1, 417 (1960).

[6] A. K. Bhatia and A. Temkin, Rev. Mod. Phys. 36, 1050 (1964); S. I. Nikitin and V. N. Ostrovsky, J. Phys. B 18, 4349 (1985).

[7] A. V. Matveenko, Phys. Rev. A 59, 1034 (1999).

[8] N. L. Manakov, S. I. Marmo, and A. V. Meremianin, J. Phys. B 29, 2711 (1996).

[9] J. S. Briggs and V. Schmidt, J. Phys. B 33, R1 (2000).

[10] A. V. Bezverbnyi, Zh. Exp. Teor. Fiz. 118, 1066 (2000) [Sov. Phys. JETP 91, 921 (2000)].
[11] H. E. Moses, Nuovo Cimento A 40, 1120 (1965); Ann. Phys. (N.Y.) 37, 224 (1966); 42, 343 (1967).

[12] M. S. Marinov, Yad. Fiz. 5, 1321 (1967) [Sov. J. Nucl. Phys. 5, 943 (1967)].

[13] N. L. Manakov and A. V. Meremianin, Zh. Exp. Teor. Fiz. 111, 1984 (1997) [Sov. Phys. JETP 84, 1080 (1997)].

[14] A. V. Taichenachev, A. V. Tumaikin, and V. I. Yudin, Zh. Exp. Teor. Fiz. 118, 77 (2000) [Sov. Phys. JETP 91, 67 (2000)].

[15] A. Erdelyi, W. Magnus, F. Oberhettinger, and F. G. Tricomi, Higher Trancendental Functions, Bateman Manuscript Project Vol. II (McGraw-Hill, New York, 1955).

[16] W. Happer, Ann. Phys. (N.Y.) 48, 579 (1968).

[17] L. C. Biedenharn and J. D. Louck, Angular Momentum in Quantum Physics: Theory and Application (Addison-Wesley, Reading, MA, 1981).

[18] E. W. Hobson, The Theory of Spherical and Ellipsoidal Harmonics (Cambridge University Press, Cambridge, England, 1931), Chap. IV, Sec. 79, p. 126. 